\title{
IMAGE DISTORTION ANALYSIS AND CLASSIFICATION SCHEME USING A NEURAL APPROACH
}

\author{
Aladine Chetouani ${ }^{1}$, Azeddine Beghdadi ${ }^{1}$, Mohamed Deriche ${ }^{2}$ \\ ${ }^{1}$ Laboratoire de Traitement et de Transport de l'Information, Institut Galilée - Université Paris 13 \\ ${ }^{2}$ King Fahd University of Petroleum \& Minerals, EE Dept., Saudi Arabia \\ aladine.chetouani@univ-paris13.fr, azeddine.beghdadi@univ-paris13.fr, mderiche@kfupm.edu.sa
}

\begin{abstract}
Generally, IQMs are not able to well predict the image quality for all degradations. Indeed, well performance could be obtained for a given degradation and poor results for others. This is essentially due to the fact that the efficiency of IQMs depends highly on the degradation specificity. To overcome this limitation, we propose to first identify the type of degradation before measuring the quality of the image. The degradation classification is performed using an Artificial Neural Networks (ANN). Then, the most appropriate IQM could be used to estimate the image quality. The obtained results in terms of classification prove the efficiency of the proposed method. This scheme could provide a powerful image distortions recognition and classification that could be used in a image quality assessment system.
\end{abstract}

Index Terms - Image Quality, Classification, Artificial Neural Networks, Degradations

\section{INTRODUCTION}

A lot of Image Quality Measures (IQMs) has been proposed in literature. PSNR (Peak Signal to Noise Ratio) is one of the most known metric. However, PSNR provides poor results in terms of correlation with subjective measures such as the Mean Opinion Score (MOS). Some methods were proposed to improve the PSNR by adding Human Visual System (HVS) characteristics such as the PNSR-HVS [3]. This metric takes into account the limitations of the HVS in discerning fine details in an image. This limitation is simulated using the Contrast Sensitivity Function (CSF) [4]. A more recent version was developed which takes into account masking effects [5] in the DCT domain [6]. More complex IQMs inspired only on HVS models have been developed such as the VDP [7] by exploiting the CSF and a multi-channel decomposition scheme [8]. Other metrics, such as the SSIM [9], use structural characteristics, or mutual information concepts [10] to quantify image degradation.
Despite all of these proposed methods provide generally, well results for a given degradation and worst performance for some others. This is due to the fact that the efficiency of the Full Reference (FR) metrics depends highly on the specificity of the degradation and also the image content. To overcome this limitation, we propose to first identify the type of degradation before measuring its quality. Our work is focused on the most common artifact.

To identify the type of the degradation, we propose to use an Artificial Neural Networks (ANN) which is used to model the degradation in image.

The proposed scheme could provide a powerful image distortion classification and image quality analysis tool which could be used in an adaptive image quality assessment system. The potential benefits and limitations of this approach are evaluated in terms of classification errors and identification.

The paper is organized as follows: Section 2 describes the proposed method and the image database used in our experiments. Our experimental results are discussed in section 3 followed by some concluding remarks in section 4 .

\section{PROPOSED METHOD}

The aim of this study is to propose a new scheme to measure the objective quality in image. We propose first to detect the type of the degradation of a given image and then we measure the quality using the more appropriate IQM.

To more explicit the importance to know the type of the degradation before measuring the image quality, we propose to do a simple test for two typical degradations: blur and JPEG. For each type of degradation, we ranked the different IQMs using the Pearson's Correlation Coefficient (PCC). The IQMs use here are the following: VIF, VIFP [10], VSNR [12], PSNR-HVS (PSNRH) [3], PSNRHVS-M (PSNRM) [6], SSIM [9], NQM [13], UQI [14], XYZ [15], IFC [16], WSNR [17] and SNR. 
Table 1 shows the obtained ranking. It can be noticed that for blur, VIFP provides the best results. However, for JPEG compression, PSNRH provides better results than VIFP.

Table 1. IQM ranking for blur and JPEG distortions

\begin{tabular}{|c|c|c|}
\hline IQM ranking & \multicolumn{2}{|c|}{ Degradation type } \\
\hline & Blur (5) & JPEG (7) \\
\hline $\mathbf{1}$ & VIFP & PSNRH \\
\hline $\mathbf{2}$ & VIF & PSNRM \\
\hline $\mathbf{3}$ & WSNR & VIF \\
\hline $\mathbf{4}$ & VSNR & WSNR \\
\hline $\mathbf{5}$ & PSNRM & NQM \\
\hline $\mathbf{6}$ & PSNRH & VIFP \\
\hline $\mathbf{7}$ & SSIM & VSNR \\
\hline $\mathbf{8}$ & UQI & SSIM \\
\hline $\mathbf{9}$ & NQM & XYZ \\
\hline $\mathbf{1 0}$ & IFC & SNR \\
\hline $\mathbf{1 1}$ & SNR & UQI \\
\hline $\mathbf{1 2}$ & XYZ & IFC \\
\hline
\end{tabular}

Table 2 presents the PCCs for the 2 types of degradations. This result confirms our remarks. Indeed, a given IQM can better quantify the quality of a given image than others, under the degradation type.

Table 2. PCC for blur \& JPEG distortions.

\begin{tabular}{|c|c|c|}
\hline \multirow{2}{*}{ Degradation type } & \multicolumn{2}{|c|}{ PCC } \\
\cline { 2 - 3 } & VIFP & PSNRH \\
\hline JPEG & $\mathbf{0 . 9 4 1 6}$ & 0.9120 \\
\hline Blur & 0.9188 & $\mathbf{0 . 9 5 4 3}$ \\
\hline
\end{tabular}

In this section, the image database used is first presented. Then, the feature extraction and the modeling process is described. The flowchart of the proposed method is presented in Fig.1.

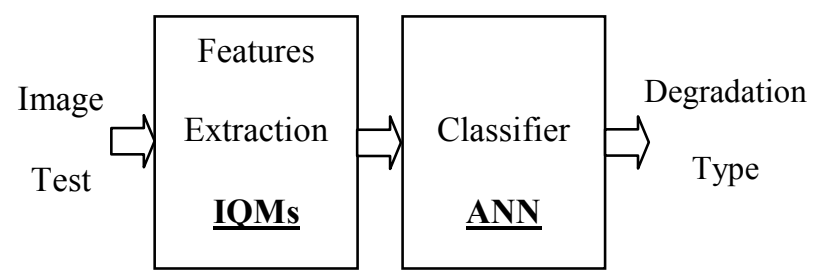

Figure 1. Flowchart of the proposed method.

\subsection{Image database description}

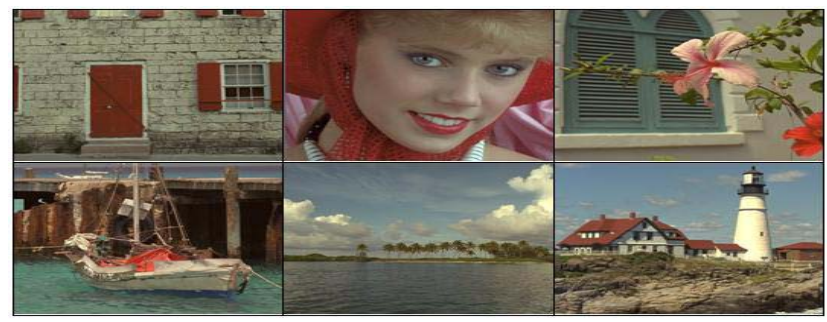

Figure 2. Some original images of the TID database.

Different image databases are available. Here, the TID 2008 database is used [9]. TID 2008 contains 17 types of degradations using 25 original images. For each degradation, 100 images are available (4 degradation levels per image, i.e. $25 * 4$ images).

Some of them are presented in Fig.2. In this study, we consider only 8 degradations referred by the authors as the most common artifacts. Table 3 lists the considered degradation types.

Table 3. Considered degradation types

\begin{tabular}{|c|l|}
\hline Degradation & \multicolumn{1}{|c|}{ Type } \\
\hline $\mathbf{1}$ & Additive Gaussian noise \\
\hline $\mathbf{2}$ & Spatially correlated noise \\
\hline $\mathbf{3}$ & Impulse noise \\
\hline $\mathbf{4}$ & Quantization noise \\
\hline $\mathbf{5}$ & Gaussian blur \\
\hline $\mathbf{6}$ & Image denoising \\
\hline $\mathbf{7}$ & JPEG compression \\
\hline $\mathbf{8}$ & JPEG2000 compression \\
\hline
\end{tabular}

\subsection{Features extraction}

As features, we propose to use the most known IQMs, cited above. Note that we assume that we have access to the original and the degraded images. Some of the used IQMs are HVS-based (such as the PSNRHVS) while others are based on pixel wise differences (MSE-based metrics) or local structural information like the SSIM.

\subsection{Classification process}

Once the features are obtained, Artificial Neural Network is used to detect the degradation type. In particular, we treat various degradations as different classes in the classifier. The IQMs estimated from a given image are seen as the extracted feature vector. The ANN used here is a Multi Layer Perceptron (MLP) (see Fig.3). The output value $x_{n}^{c}$ of a given neuron $n$ in the layer $c$ is given by: 


$$
\begin{gathered}
x_{n}^{c}=f\left(\sum_{u=0}^{N} \omega_{n u}^{c-1}{ }_{n}^{c} x_{u}^{c-1}\right) \\
\text { w ith } f(V)=\frac{e^{2 V}-1}{e^{2 V}+1}
\end{gathered}
$$

where $N_{c}$ denotes the number of neurons in layer $c . \omega_{n j}^{c}$ is the weight of the neuron $n . x_{u}^{c-1}$ is the value of the neuron $u$ in layer c-1. $f(V)$ is the activation function.

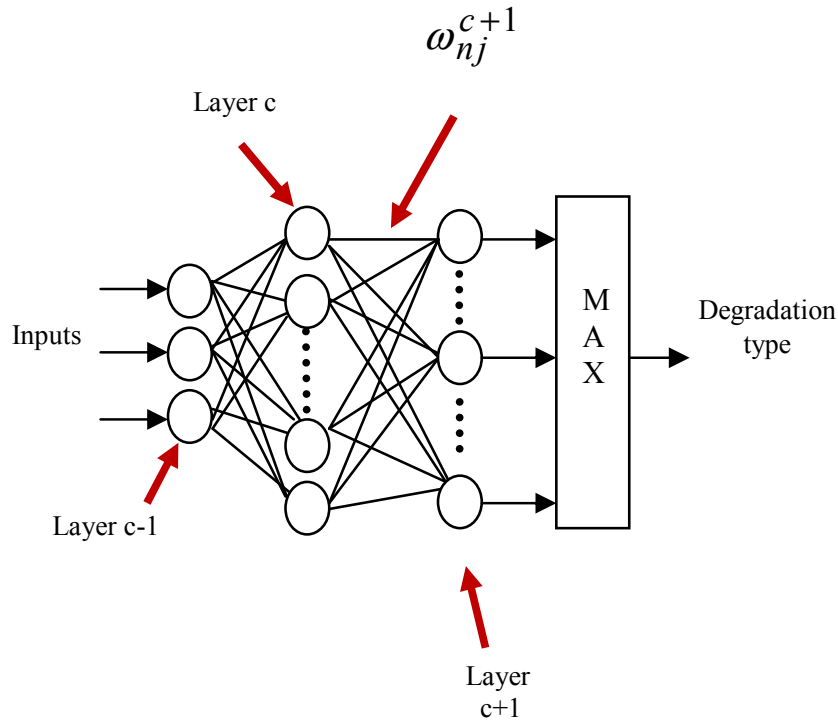

Figure 3. The Artificial Neural Network considered.

For a given degradation, the number of input neurons is equal to the number of selected IQMs (here 12). The output layer is formed by $\mathrm{N}$ neurons where $\mathrm{N}$ denotes the number of classes (i.e. number of degradations, here 8). Note that in this study, we propose to use only one ANN. Thus, in the learning phase, for a given degradation, the target values are equal to 1 for the neuron which corresponds the selected degradation and 0 for other neurons. The input values are first scaled to the range $[-1,+1]$. This range is due the sigmoid function used as for activation. This sigmoid function has been previously used by the Visual Quality Experts Group (VQEG) [18].

During the learning phase, we first divide the used image database in 2 sets:

- $\quad$ Training set: used only in the learning stage. Around 50 images per degradation.

- Test set: used only to evaluate the performance of the proposed method. Around 25 images per degradation.
In the learning phase, the back propagation method is used to estimate the parameters of the ANN. Finally, the structure of the ANN model is given below:

- Input: 12 (i.e. considered IQMs, the index values are scaled to the range $[-1,+1])$.

- Hidden Layer: 1 (the number of neurons is equal to 19).

- Output: 8 (i.e. number of degradations).

\section{RESULTS AND DISCUSSION}

The efficiency of the proposed method is tested in terms of classification rate using only the test set which does not contain any image used in the learning process. The percentage of good classification for each degradation is presented in Fig.4.

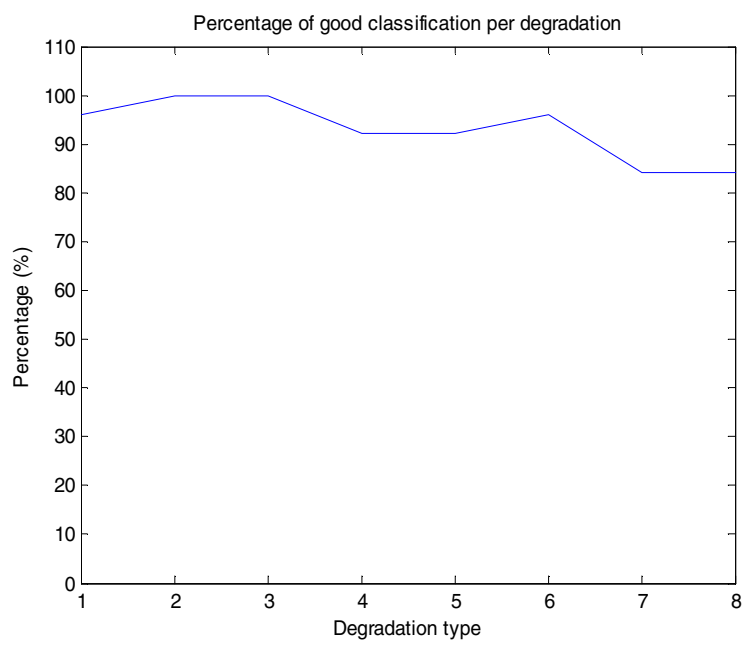

Figure 4. Percentage of good classification per degradation.

The mean percentage of correct classification, for all considered degradations, is equal to $93 \%$. The lowest value is obtained for the JPEG and JPEG 2000 degradation (84\% of correct classification).

The resulting confusion matrix is shown in Fig.5. It could be noticed that the classifier process is efficient in detecting the degradation types. For some degradations, a 100\% of correct classification rate is obtained. 
Estimated Class

\begin{tabular}{|c|c|c|c|c|c|c|c|c|}
\hline & 1 & 2 & 3 & 4 & 5 & 6 & 7 & 8 \\
\hline 1 & 96 & 4 & 0 & 0 & 0 & 0 & 0 & 0 \\
\hline 2 & 0 & 100 & 0 & 0 & 0 & 0 & 0 & 0 \\
\hline 3 & 0 & 0 & 100 & 0 & 0 & 0 & 0 & 0 \\
\hline 4 & 4 & 0 & 4 & 92 & 0 & 0 & 0 & 0 \\
\hline 5 & 0 & 0 & 0 & 0 & 92 & 8 & 0 & 0 \\
\hline 6 & 0 & 0 & 0 & 0 & 4 & 96 & 0 & 0 \\
\hline 7 & 0 & 0 & 0 & 0 & 0 & 8 & 84 & 8 \\
\hline 8 & 0 & 0 & 0 & 0 & 4 & 12 & 0 & 84 \\
\hline
\end{tabular}

Figure 5. Confusion matrix.

\section{CONCLUSION}

In this study, an image distortion analysis and classification scheme based on neural networks is proposed for image quality assessment. It is shown that the ANN-based classifier is robust and could be used to determine efficiently the type of the degradation in an image. The results are promising and open a new perspective for image quality assessment.

The next step is to use this classification scheme in the design of an adapted image quality assessment system. The introduction of feature selection scheme could be used in order to increase the performance of the proposed method. This will be done in a future work.

\section{REFERENCES}

[1] M.C.Q. Farias, "No-Reference and Reduced Reference Video Quality Metrics: New Contributions", Ph.D Dissertation, University of California, Santa Barbara, 2004.

[2] H.R. Wu and K.R. Rao, "Digital Video Image Quality and Perceptual Coding", CRC Press (ISBN: 0-8247-2777-0), 2005.

[3] K. Egiazarian, J. Astola, N. Ponomarenko, V. Lukin, F. Battisti and M. Carli, "New full-reference quality metrics based on HVS", CD-ROM Proceedings of the Second International Workshop on VPQM, http://www.vpqm.org/, 2006.

[4] A.B. Watson, H.B. Barlow and J.G. Robson, "What does the eye see best?", Nature, pp. 419-422, 1983.

[5] G. Legge and J. Foley, "Contrast masking in human vision", Vol.70, pp. 1458-4471, 1980.

[6] N. Ponomarenko, F. Silvestri, K. Egiazarian, M. Carli, J. Astola and V. Lukin, "On between-coefficient contrast masking of DCT basis functions", CD-ROM Proceedings of the Third International Workshop on VPQM, http://www.vpqm.org/, 2007.

[7] S. Daly, "The Visible Differences Predictor: An Algorithm for the Assessment of Image Fidelity", Digital Images and Human Vision, Watson A.B.(Ed.). MIT Press, ch14, pp. 179206, 1993.

[8] A.B.Watson, "The cortex transform: Rapid computation of simulated neural images," Computer Vision Graphics and Image Processing, pp .311-327, 1987.

[9] Z. Wang, E.P. Simoncelli and A.C Bovik, "Multi-scale structural similarity for image quality assessment", IEEE Asilomar Conference on Signals, Systems and Computers, 2003.

[10] H.R. Sheikh and A.C Bovik, "Image information and visual quality", IEEE Transactions on Image Processing, Vol.15, no.2, pp. 430-444, 2006.

[11] N. Ponomarenko, M. Carli, V. Lukin, K. Egiazarian, J. Astola and F. Battisti, "Color Image Database for Evaluation of Image Quality Metrics", Proceedings of International Workshop on Multimedia Signal Processing, Australia, pp. 403-408, 2008.

[12] D.M. Chandler and S.S. Hemami, "VSNR: A Wavelet-Based Visual Signal-to-Noise Ratio for Natural Images", IEEE Transactions on Image Proc., Vol. 16, pp. 2284-2298, 2007.

[13] N. Damera-Venkata, T. Kite, W. Geisler, B. Evans and A.C. Bovik, "Image Quality Assessment Based on a Degradation Model", IEEE Transactions on Image Processing, Vol. 9, pp. 636-650, 2000.

[14] Z. Wang and A.C. Bovik, "A universal image quality index", IEEE Signal Processing Letters, Vol. 9, pp. 81-84, 2002.

[15] B. W. Kolpatzik and C. A. Bouman, "Optimized Universal Color Palette Design for Error Diffusion”, Journal Electronic Imaging, Vol. 4, pp. 131-143, 1995.

[16] H.R. Sheikh, A.C. Bovik and G. de Veciana, "An information fidelity criterion for image quality assessment using natural scene statistics", IEEE Transactions on Image Processing, Vol.14, no.12, pp. 2117-2128, 2005.

[17] T. Mitsa and K. Varkur, "Evaluation of contrast sensitivity functions for the formulation of quality measures incorporated in halftoning algorithms", IEEE-ICASSP, pp. 301-304, 1993.

[18] G.G. Judge, R. C. Hill, W. E. Griffiths, H. Lutkepohl, and T.-C. Lee, "Introduction to the Theory and Practice of Econometrics", Wiley (ISBN 0-4716-2414-4), 1988. 